were practiced as followed: ten portable pools based at the primary schools, one community pool and two beach locations. Training materials were revised and translated from Royal Life Saving Society Australia (RLSSA) documents which can be applied on local situation and physical strength of Vietnamese children. 120 instructors and 8 lifeguards were recruited and trained that included 19 RLSSA certified instructors. After 3 months program, total 4718 children participated in the certification test with requirements of continuously swimming 25 meters and floating for $90 \mathrm{~s}$, demonstrating basic knowledge of water safety and, for children over 10 years old, basic Cardiopulmonary Resuscitation techniques. The number of boys participated as much as girls, highest numbers are focused on 9 and 10 years old. $25 \mathrm{~m}$ swimming and 90 s floating capacity were in direct ratio with ages and attendances. SwimSafe program was touched the success. Lots of worthy experiences learnt could be applied to other communities in Vietnam.

\section{TRAINING SURVIVAL SWIM TO PREVENT DROWNING FOR CHILDREN AT PRIMARY SCHOOL IN DA NANG CITY, SUMMER 2009}

V Nguyen*, C Pham, M Linnan Correspondence: Hanoi School of Public Health, 138 Giang Vo street, Ba Dinh district, Hanoi city, +84 , Vietnam

10.1136/ip.2010.029215.509

Drowning is a global public health problem. In Vietnam, according to Vietnam Multi-centre Injury Survey, drowning was the leading killer of children under 18 years old with the rate of 22.6/100 000 annually. The drowning rate of children is 10 times bigger than developed country in comparison. Researches gave evident that training on survival swim could be the most achieved method to reduce risks of children drowning. The SwimSafe program aims to train 5000 children from Da nang 20 lessons of survival swimming and water safety knowledge in 3 months summer 2009. Three kinds of model 